\title{
Conservation of Spatial Memory Function in the Pallial Forebrain of Reptiles and Ray-Finned Fishes
}

\author{
Fernando Rodríguez, J. Carlos López, J. Pedro Vargas, Yolanda Gómez, Cristina Broglio, and Cosme Salas \\ Laboratorio de Psicobiología, Universidad de Sevilla, 41005 Seville, Spain
}

The hippocampus of mammals and birds is critical for spatial memory. Neuroanatomical evidence indicates that the medial cortex (MC) of reptiles and the lateral pallium (LP) of ray-finned fishes could be homologous to the hippocampus of mammals and birds. In this work, we studied the effects of lesions to the MC of turtles and to the LP of goldfish in spatial memory. Lesioned animals were trained in place, and cue maze tasks and crucial probe and transfer tests were performed. In experiment 1, MC-lesioned turtles in the place task failed to locate the goal during trials in which new start positions were used, whereas sham animals navigated directly to the goal independently of start location. In contrast, no deficit was observed in cue learning. In experiment 2, LP lesion produced a dramatic impairment in goldfish trained in the place task, whereas medial and dorsal pallium lesions did not decrease accuracy. In addi- tion, none of these pallial lesions produced deficits in cue learning. These results indicate that lesions to the MC of turtles and to the LP of goldfish, like hippocampal lesions in mammals and birds, selectively impair map-like memory representations of the environmental space. Thus, the forebrain structures of reptiles and teleost fish neuroanatomically equivalent to the mammalian and avian hippocampus also share a central role in spatial cognition. Present results suggest that the presence of a hippocampus-dependent spatial memory system is a primitive feature of the vertebrate forebrain that has been conserved through evolution.

Key words: spatial memory; place learning; pallial forebrain; hippocampus; medial cortex; hippocampal pallium; brain evolution; reptiles; turtles; teleost fish; goldfish; amniotes; rayfinned fishes
The vertebrate forebrain shows an impressive range of adaptation and morphological variation among species (Northcutt, 1995; Butler and Hodos, 1996; Nieuwenhuys et al., 1998). One major variation is found in the telencephalon of the actinopterygian fish (for instance, teleost fish), which undergoes a process of eversion during embryonic development, relative to the telencephalon of other vertebrates (for instance, amniotes), which develops by a process of evagination (Nieuwenhuys, 1963; Northcutt and Braford, 1980) (see Fig. 1). These different developmental processes produce notable morphological divergence, mainly, paired telencephalic hemispheres with internal ventricles in nonactinopterygians, which contrast with the massive telencephalic hemispheres flanking a single ventricular cavity in the actinopterygian radiation. Nonetheless, neuroanatomical findings reveal that the telencephalon of the actinopterygian and the nonactinopterygian vertebrates shows a comparable basic pattern of organization. As in amniotes, the telencephalon of ray-finned fishes consists of a dorsally located pallial mantle and subpallial regions (Northcutt, 1981). In addition, increasing neuroanatomical evidence indicates that the main subdivisions of the pallium in the actinopterygian telencephalon may be homologous to various pallial areas in amniotes, including a region possibly homologous

\footnotetext{
Received Nov. 15, 2001; revised Jan. 7, 2002; accepted Jan. 9, 2002.

This research was supported by grants from the Spanish Direccion General de Enseñanza Superior and Junta de Andalucía. We thank V. P. Bingman, A. Butler, H. Eichenbaum, L. Nadel, R. Nieuwenhuys, R. G. Northcutt, J. B. Overmier, A. Reiner, and $\mathrm{C}$. Thinus-Blanc for advice and critical reading of an earlier version of this manuscript. We thank G. Labrador for technical assistance.

Correspondence should be addressed to Cosme Salas, Laboratorio de Psicobiología, Universidad de Sevilla, Campus Santiago Ramón y Cajal, Avenida San Francisco Javier, 41005 Seville, Spain. E-mail: cosme@us.es.

Copyright (C) 2002 Society for Neuroscience $0270-6474 / 02 / 222894-\bullet \$ 15.00 / 0$
}

to the hippocampal pallium (Northcutt and Braford, 1980; Nieuwenhuys and Meek, 1990; Braford, 1995; Northcutt, 1995; Butler, 2000; Vargas et al., 2000).

In mammals and birds, the hippocampus is critical for map-like or relational memory representations of allocentric space (O'Keefe and Nadel, 1978; Sherry and Duff, 1996; Bingman et al., 1998; Burgess et al., 1999; Eichenbaum et al., 1999). In these vertebrate groups, lesions to the hippocampal formation produce selective impairments in spatial tasks that require the encoding of relationships among multiple environmental features (place learning) but not in tasks that require approaching a single cue or simple nonspatial discriminations (Morris et al., 1982; Gaffan and Harrison, 1989; Sherry and Vaccarino, 1989; Fremouw et al., 1997). Although spatial capabilities similar to those described in mammals and birds have been described recently also in reptiles (López et al., 2000c, 2001) and teleost fish (Rodríguez et al., 1994; López et al., 1999, 2000a), data regarding the neural mechanisms underlying spatial cognition in vertebrates other than mammals and birds are still primarily lacking. For the present work, we selected a species representative of the amniote (evaginated) forebrain pattern, the painted turtle, and another representative species possessing the actinopterygian (everted) forebrain pattern, the goldfish, to investigate whether the reptilian medial cortex (MC) and the teleost lateral telencephalic pallium, both proposed as homologs to the hippocampus on the basis of neuroanatomical evidence, are selectively involved in spatial memory. With this aim, in a first experiment, we evaluated the effects of lesions to the medial cortex of turtles in place and cue maze procedures. In a second experiment, we analyzed the effects of lesions to the lateral (LP), dorsal (DP), or medial (MP) pallium of goldfish in similar place and cue tasks. 


\section{MATERIALS AND METHODS \\ Experiment 1: spatial memory deficits after medial cortex lesion in turtles}

The reptilian medial cortex is considered homologous to the hippocampal formation of mammals and birds on the basis of anatomical and physiological evidence (Northcutt, 1981; Schwerdtfeger and Smeets, 1988; Ulinski, 1990; Muñoz et al., 1998; Nieuwenhuys et al., 1998). In addition, turtles, like mammals and birds, have place-memory capabilities, based on map-like or relational memory representations of the allocentric space. For example, turtles trained in standard place tasks were able to navigate with accuracy to the goal from unfamiliar start points, adopting spontaneously novel routes to the goal, and their performance was also resistant to a partial loss of relevant environmental information (López et al., 2000c, 2001). In mammals and birds, these spatial cognition capabilities are based on the hippocampal function (O'Keefe and Nadel, 1978; Sherry and Duff, 1996; Bingman et al., 1998; Burgess et al., 1999). Thus, if the cognitive mapping capabilities herein described in turtles are homologous to those described in mammals and birds, they should be based on the medial cortex function. To test this possibility, medial cortex-lesioned and sham-operated (Sh) turtles were trained in place, cue, or control maze procedures. Also, probe and transfer tests were performed to analyze the spatial strategies used by each group to solve their respective tasks.

\section{Subjects}

The subjects were 48 painted turtles (Pseudemys scripta), 10-12 $\mathrm{cm}$ in shell length. For 2 months before the experiments, turtles were housed in small groups in 2001 glass aquaria containing a $20 \times 20 \mathrm{~cm}$ dry platform, with aerated filtered water $\left(24 \pm 1^{\circ} \mathrm{C}\right)$. Animals were kept on a $14 / 10 \mathrm{hr}$ light/dark cycle and were fed twice per day. Two days before the experiment, the animals were deprived of any food. Throughout the experiment, the animals consumed only the food sticks they obtained in the daily session. Each stick consisted of $\sim 20 \mathrm{mg}$ of dry food (TetraPond; Ulrich Baemsch GmbH, Melle, Germany). Use and handling of the animals were under the guidelines established by Directive 86/609/CEE of the European Community Council and the Spanish Real Decreto 223/1988.

\section{Apparatus}

The apparatus, experimental room, and general procedures were similar to those used in previous studies (Rodríguez et al., 1994; Salas et al., 1996b; López et al., 2000a,c). Briefly, the apparatus was an elevated four-arm maze (each arm was 75-cm-long) made out of Perspex, with transparent walls and a white opaque floor. Only three of the arms were used for the training and probe trials, whereas the four arms of the maze were used during the transfer trials (TTs). For each trial, the start position was blocked off by a 20 -cm-high opaque Perspex guillotine door placed $16 \mathrm{~cm}$ from an arm end and controlled at a distance by a hand-operated device. The access to the arm not used in each training trial was blocked by means of a $15-\mathrm{cm}$-high removable barrier. Before every session, the maze was filled with aerated and filtered water at $24 \pm$ $1^{\circ} \mathrm{C}$ to a depth of $3 \mathrm{~cm}$ to facilitate the displacements of the animal. The maze was placed, at a height of $50 \mathrm{~cm}$, in the center of a room measuring $4.8 \times 5 \times 3.1 \mathrm{~m}$ that presented abundant distal visual cues distributed all around. To exclude the possible use of uncontrolled intramaze cues, the maze was randomly rotated between the experimental sessions. When necessary, the maze could be surrounded by a gray curtain that hung from ceiling to floor, thus excluding the use of the extramaze visual cues.

For each training trial, removable feeders were fixed to the floor of the maze at a distance of $5 \mathrm{~cm}$ from the end of each of the two accessible arms. The goal arm was the one containing the baited feeder. These feeders consisted of 4-cm-high dark food cups presenting a 1.5-cm-deep cavity at the top, in which a food stick could be hidden. The food was not visible, but the turtle removed it by lifting the head over the feeder.

During the trials, the observer remained in an adjacent enclosure, from which he controlled the guillotine doors of the maze and observed the behavior of the turtles through a small opening.

\section{Behavioral procedure}

Pretraining. Before the experiment, turtles were pretrained during 2 consecutive days to obtain food from the feeder. With this aim, the turtles were individually placed in a small aquarium $(40 \times 30 \times 30 \mathrm{~cm})$ provided with a food cup, identical to those to be used during training trials, that was successively baited with a food pellet until the subject consumed three pellets. This aquarium was placed in a room adjoining the experimental room. These sessions were followed by $2 \mathrm{hr}$ preexposure sessions, in which each subject was individually placed in the experimental room and allowed to freely explore the maze. The food holders and the barriers were removed from the maze during these sessions. After the pretraining period, the animals were deprived of any food for $2 \mathrm{~d}$ before the experiment proper.

Training. Turtles were randomly assigned to one of the following experimental training procedures: place task, cue task, and control task. One-half of the place and cue animals had MC lesion and the other one-half were Sh, whereas the control group animals were all unoperated. The final distribution of the turtles in the different groups was as follows: in the place task, MC, $n=9$ and Sh, $n=10$; in the cue task, MC, $n=9$ and Sh, $n=10$; and in the control task, $n=10$.

Place task. In this task, two opposite start positions were used in a pseudorandom order (southwest arm, 50\%; northeast arm, 50\%), but the goal arm remained always in the same place in the room throughout the experiment. No fixed-turn strategy was adequate to solve this task, given that the animals were required to make a left or right turn, depending on the start position. The location of the goal was counterbalanced within the turtles trained in this procedure, so that one-half of the animals were trained to obtain the reward in the northwest arm and the remaining animals obtained the reward on the southeast arm.

Cue task. In this task, the turtles always obtained the reward in the arm presenting a conspicuous intramaze visual cue consisting of a red removable panel $(14 \times 22 \mathrm{~cm})$, fixed at the end of the goal arm. For each training trial, goal location was assigned in a pseudorandom order between the two possible positions ( $50 \%$ each), so extramaze cues were irrelevant to task solution. Thus, in this procedure, the goal could be found by reference to an intramaze beacon but not to more distant landmarks. In addition, two opposite start arms were used pseudorandomly (50\% each).

Control task. In this task, the animals were trained to find reward in two possible unsignaled goal locations that varied in a pseudorandom order across trials (50\% each). Also, the animals in this group were released pseudorandomly from either of two opposite start arms $(50 \%$ each) for each trial. This procedure was designed to control the possibility that the animals could find the reward by attending to odor traces or other uncontrolled variables.

The turtles were individually trained in daily three-trial sessions. To begin each trial, the animal was carefully placed in the start box of the maze and confined there for $15 \mathrm{sec}$. Then, the guillotine door was raised and lowered after the animal left the start box, allowing the animal to perform free displacements in the accessible arms. A choice was recorded when the animal traveled $15 \mathrm{~cm}$ into the selected maze arm. A trial was considered correct only when the first choice was correct. The subject remained in the maze until the reward had been consumed (correction procedure) or until $15 \mathrm{~min}$ had elapsed. At the end of each trial, the animal was gently removed from the maze and returned to the home aquarium for a $30 \mathrm{~min}$ intertrial interval. Error choices and time used to obtain the reward were also recorded. A learning criterion of 13 correct trials of 15 (86\% correct over five consecutive sessions) was established.

Transfer and probe tests. When the animals reached the criterion, additional postcriterion sessions were conducted, during which the nonreinforced probe and transfer trials were interspersed in a pseudorandom order between training trials. During transfer and probe trials, the feeders were removed from the maze; thus, reinforcement was not available. On any given session, only one transfer or probe trial was performed, and at least three training trials were conducted between either two probe or transfer trials.

Transfer trials. Different TTs in which the animals started from novel start positions were conducted. These trials were designed to study whether the turtles trained in the place procedure solved the task on the basis of place strategies, i.e., to study whether the animals show the capability to reach the goal regardless of start position, and whether they are able to adopt novel routes to the goal. In these transfer trials, the maze was displaced within the room in such a way that novel start locations, never used during training, were used. In each TT, the end of one of the arms occupied the same place in the room in which the animals obtained the reward during training trials. For the turtles in the cue procedure, the TTs were similar to those used in the place procedure, except that the intramaze cue signaling the baited arm during training trials was maintained. In the cue procedure, TTs were conducted to test 
whether the turtles solved the task by means of a guidance strategy, approaching the intramaze cue directly associated to the goal. During TTs, all of the maze arms remained opened, enabling the animals to choose freely among all of them (so, in these trials, chance level was $33.3 \%$ ). For each animal, 18 TTs were conducted.

Probe trials. Three types of probe trials, consisting of the complete or partial exclusion of the visual cues, were conducted. These tests were aimed to determine the relevance of the experimental visual cues for the $\mathrm{MC}$ and Sh turtles trained in the place and the cue tasks. Probe trials type 1 (PT1) consisted of eliminating every extramaze cue by surrounding the entire maze with a gray curtain. In probe trials type 2 (PT2) and type 3 (PT3), the curtains concealed only one-half of the extramaze distal cues, whereas the other one-half of the experimental room remained uncovered. In PT2, only those extramaze cues placed in the proximity of the goal were occluded, and, in PT3, the most distant extramaze visual cues relative to goal location were excluded. For the turtles trained in the cue task, the intramaze visual cue that signaled directly the baited feeder during training trials was maintained. An additional probe test (PT4) consisting of the removal of the single intramaze visual cue associated to the goal was conducted for the turtles trained in the cue task. Similar to training trials, two start positions (50\% each one) were used randomly during probe trials. Four probe trials of each type were conducted for each animal.

Surgery. Turtles were anesthetized with tricaine methanesulfonate (MS222) $(0.6 \mathrm{mg} / \mathrm{kg}$ body weight; Sigma, St. Louis, MO) and placed in a stereotaxic apparatus. The scalp was surgically cleaned, incised, and retracted, and 10 small burr holes $(0.5 \mathrm{~mm}$ in diameter $)$ were drilled in the skull for bilateral penetrations of electrodes. Xilocaine anesthetic was administered along the incision borders. Electrolytic lesions were produced delivering $0.5 \mathrm{~mA}$ for $30 \mathrm{sec}$, by monopolar electrodes stereotaxically placed at the following coordinates in each hemisphere (anterior, ventral, and lateral, respectively): $0.4,2$, and 1.4; 1.4, 2, and 1.4; $2.4,2$, and $0.7 ; 3.4,1.5$, and 0.6 ; and $4.4,1.5$, and 0.5 (corrected coordinates from the turtle brain atlas of Powers and Reiner, 1980). After surgery, the scalp was sutured. Sham operations were performed exactly in the same way except that no current was passed through the electrode. Subjects were allowed to recover for 1 week before training.

Histology. On completion of behavioral testing, turtles were perfused transcardially with a fixative solution ( $10 \%$ formalin in $0.1 \mathrm{M}$ PBS). The brains were removed from the skull, inspected for an initial evaluation of damage, sectioned at $40 \mu \mathrm{m}$ in the coronal plane, and Nissl stained for detailed histological analysis. The extent of damage was determined for each animal by reconstructing the location and extent of the lesion on the plates of the turtle brain atlas (Powers and Reiner, 1980).

\section{Experiment 2: spatial memory deficits after lateral pallium lesion in goldfish}

Like amniotes, teleost fish are able to use place strategies to navigate to a goal by means of encoding its spatial relationships with a number of landmarks in a map-like, allocentric representation that provides a stable frame of reference (Rodríguez et al., 1994; López et al., 1999, 2000a). Thus, goldfish trained in place-learning tasks are able to reach the goal even when novel start positions are used, and they have to adopt shortcuts and novel routes in absence of local cues. Moreover, goldfish can use a number of widely distributed visual cues to solve spatial tasks by encoding the whole spatial arrangement, such that none of those cues is essential by itself to locate the goal. In addition, the complete telencephalon ablation in goldfish, like hippocampal lesions in mammals and birds, produces a severe deficit in a variety of spatial tasks that require the use of mapping strategies but not in tasks requiring cue guidance (Salas et al., 1996a,b; López et al., 2000a,b). These data are particularly interesting, because they open the possibility to identify the particular pallial areas underlying spatial cognition in teleost fish. Because of the eversion process that takes place during development in teleosts (i.e., the pallial or dorsal portion of the embryonic prosencephalic wall curves laterally) (Fig. 1), the telencephalic area that occupies a topological position compatible with the amniote medial pallium or hippocampus is the lateral pallium (Northcutt and Braford, 1980; Nieuwenhuys and Meek, 1990; Nieuwenhuys et al., 1998; Butler, 2000). In the present experiment, we studied the effects of selective pallial lesions on goldfish spatial memory by means of a place-learning procedure similar to that described for experiment 1 .

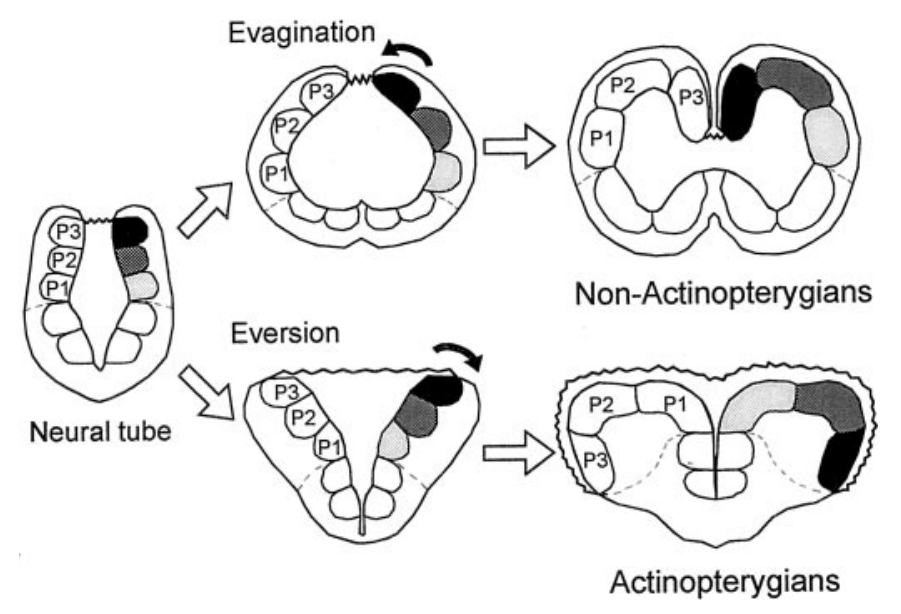

Figure 1. Schematic representation of the process of evagination and inversion that occurs in the telencephalon of non-actinopterygian vertebrates during embryonic development compared with the process of eversion or bending outward that occurs in actinopterygians. In tetrapods and many fishes, the evagination of the dorsolateral prosencephalic wall produces paired telencephalic hemispheres with internal ventricles. In actinopterygian fish, in contrast, the pallial or dorsal portion of the embryonic prosencephalic wall curves laterally, producing two massive hemispheres flanking a single ventricular cavity. $P 1, P 2$, and $P 3$ correspond to the three main subdivisions of the pallium.

\section{Subjects}

The subjects were 55 goldfish (Carassius auratus) $12-14 \mathrm{~cm}$ in length. For 2 months before the experiments, goldfish were housed in small groups in 2001 glass aquaria with aerated filtered water $\left(20 \pm 1^{\circ} \mathrm{C}\right)$. Animals were kept on a 14/10 hr light/dark cycle and were fed twice per day. Two days before the experiment, the goldfish were deprived of any food. Throughout the experiment, the animals consumed only the food sticks they obtained during the training session. Each stick consisted of $\sim 20 \mathrm{mg}$ of dry food (TetraPond; Ulrich Baemsch GmbH).

\section{Apparatus}

The apparatus and experimental room were the same used in experiment 1 , but the maze was filled with aerated and filtered water at $20 \pm 1^{\circ} \mathrm{C}$ to a depth of $15 \mathrm{~cm}$. In this experiment, the feeders consisted of removable curved glass bars (5-cm-high) with a dark latex tube inserted at the end, which could hold one stick of food. This food was not visible, and the fish took it out by suction. The feeders were fixed to the floor of the maze at a distance of $5 \mathrm{~cm}$ from the end of each of the two accessible arms. The goal arm was the one containing the baited feeder.

\section{Behavioral procedure}

Pretraining. Before the experiment, goldfish were pretrained during 2 consecutive days to obtain food from an experimental feeder that was rebaited until the fish consumed five pellets. The feeder was placed in an aquarium $(40 \times 30 \times 30 \mathrm{~cm})$ located in a room adjoining the experimental room. After this, the fish were placed in the experimental room for free maze exploration during $2 \mathrm{hr}$. After this pretraining period, the goldfish were deprived of any food for $2 \mathrm{~d}$ before the experiment proper.

Place and control tasks. The general training procedure was the same described for experiment 1 . Goldfish were randomly assigned to one of the following procedures: place task $(n=47)$ or control task $(n=8)$. These tasks were the same as for experiment 1 . Animals trained in the place procedure were randomly assigned to one of the following surgical groups: lateral (LP; $n=11)$, dorsal (DP; $n=9)$, or medial (MP; $n=9$ ) pallial lesions, complete ablation of both telencephalic hemispheres (Tel; $n=10$ ), or sham operated (Sh; $n=8$ ). The animals in the control group were unoperated.

The animals were individually trained in daily five-trial sessions according to the procedure described for experiment 1 . To begin a trial, the fish was gently placed in the start box by a small net and confined there for $15 \mathrm{sec}$. Then, the guillotine door was raised (and lowered after the fish left the start box). A choice was recorded when the tail of the fish crossed the entrance of an arm. A trial was considered correct only when 
the first choice was correct. As for experiment 1, a correction procedure was used. Once the trial was finished, the animal was removed from the maze and placed into the home aquaria for a $30 \mathrm{~min}$ intertrial interval. A learning criterion of 21 correct trials of 25 (84\% correct over five consecutive sessions) was established. When the animals reached the learning criterion, 12 additional postcriterion sessions were conducted, during which the transfer trials were interspersed. Then, surgery was performed, and, after a recovery period of $5 \mathrm{~d}, 12$ additional postsurgery retraining sessions were conducted during which transfer trials were also interspersed.

Transfer tests. TTs in which the animals started from novel start positions were interspersed in a pseudorandom order, between presurgery postcriterion trials and postsurgery retraining trials. These trials were conducted in the same way as described for experiment 1 . For each animal, 24 TTs were conducted, 12 before surgery and 12 after surgery. On any given session, only one TT was performed, and at least four training trials were conducted between either two transfer trials.

Cue task. After postsurgery retraining in the place task, goldfish were trained in a cue-learning task in a different maze and room. The apparatus and procedure used in this task were similar to those used in previous studies (Salas et al., 1996a; López et al., 1999, 2000b). Briefly, goldfish were trained in daily 25 -trial sessions to exit from a box maze $(40 \times 40 \times 25 \mathrm{~cm})$ made out of dark gray polyvinyl chloride with two start boxes and two exit doors, placed in an aquarium $(1 \times 1 \mathrm{~m})$ filled with water to a depth of $20 \mathrm{~cm}$. On each trial, one of the doors was blocked by a transparent glass barrier, leaving the other as the only exit (goal). The location of the exit varied pseudorandomly (50\% each) across trials, but it was always signaled by two stripped panels of $20 \times 25 \mathrm{~cm}$ (cue) that surrounded the goal, so correct choices involved an alternation of turns. To begin each trial, the goldfish was carefully placed in one of the start compartments with free access to the maze and left there until it passed through the goal (correction procedure). An error was scored when the fish bumped against the glass barrier, and a correct choice was scored when its head passed through the exit. A trial was considered correct only when the first choice was correct. An acquisition criterion of 20 correct trials of $25(80 \%)$ was established. When the animals reached the criterion, a cue removal test was conducted. This test consisted of 10 additional trials in which the cue was removed, but all other procedural details remained unchanged. This procedure tested the reliance of the fish on the stripped panels for performance and for the possibility that the fish could detect directly the glass barrier.

Surgery. The telencephalic pallium lesions were produced by aspiration according to the methods described previously (Salas et al., 1996a,b; López et al., 2000a,b). Goldfish were anesthetized by immersion in a solution 1:20,000 of MS222 (Sigma) and then were immobilized in a surgical chamber with constant flow of aerated water through the gills. The concentration of anesthetic in the water was kept at 1:20,000 during surgery. The dorsal skin and skull were removed carefully under visual control by means of a binocular microscope, and the fatty tissue inside was aspirated to expose the brain. The sulcus lateralis, sulcus limitans telencephali, sulcus ypsiliformis, and the anterior commissure were used as anatomical references to determine the location and extension of the to-be aspirated nervous tissue. The lesions were performed by means of a glass micropipette connected to a manual vacuum system. After ablation, the piece of skull was returned to its original position, fixed with cyanocrilate glue, and covered with dental cement. Sham operations were performed exactly in the same way except that the nervous tissue was not injured. Animals were returned to their home tank after surgery and allowed a recovery period of $5 \mathrm{~d}$ before being food deprived and returned to behavioral testing.

Histology. On completion of behavioral testing, goldfish were deeply anesthetized with a solution 1:5000 of MS222 and perfused transcardially with a fixative solution ( $10 \%$ formalin in $0.1 \mathrm{M}$ PBS). The brains were removed from the skull, inspected for an initial evaluation of damage, sectioned at $40 \mu \mathrm{m}$ in the coronal plane, and Nissl stained for histological analysis. The damage was determined for each animal by reconstructing the location and extent of the lesion on the plates of a goldfish brain atlas (Peter and Gill, 1975).

\section{RESULTS}

\section{Experiment 1}

\section{Place learning and MC lesion}

Figure $2 A$ shows the learning curve of the turtles trained in the place procedure during training sessions. No significant differ-

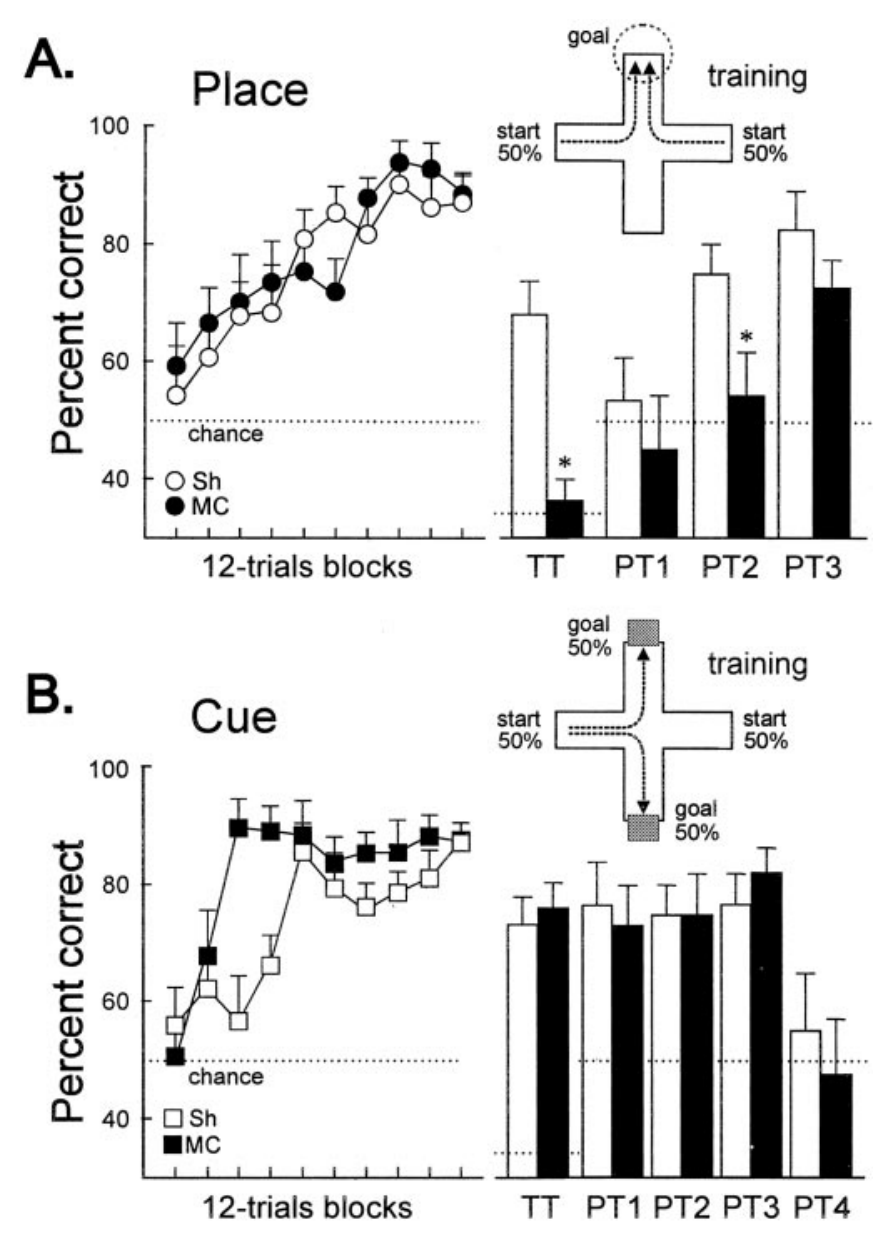

Figure 2. Spatial memory deficits after medial cortex lesion in turtles. $A$, Mean \pm SEM percentage of correct choices across training and during TTs and PTs for the turtles in the place procedure. Note that, during TTs, all of the maze arms were opened so that chance level was $33.3 \%$. B, Mean \pm SEM percentage of correct choices across training and during TTs and PTs in the cue procedure. For a description of the TTs and PTs, see Materials and Methods. The maze diagrams show the training procedure for the place and the cue tasks. White bars, Sh; black bars, MC. ${ }^{*} p<0.05$.

ences in any behavioral measure were observed between the counterbalanced conditions during training $\left(\mathrm{Sh}, F_{(1,8)}=0.20, p=\right.$ 0.66 ; $\left.\mathrm{MC}, F_{(1,7)}=0.47, p=0.83\right)$; therefore, these data were collapsed to calculate group percentages. The percentage of correct trials was near chance level during the initial sessions of the experiment. With training, both MC and Sh turtles progressively improved their accuracy until they reached the criterion. ANOVA showed that MC and Sh turtles in the place task did not differ in learning rate along training $\left(F_{(1,17)}=0.148, p=0.7\right)$ (Fig. $2 A)$. However, a significant effect of the lesion appeared during TTs, in which new start positions were used, and during probe tests (PT1, PT2, and PT3), in which extramaze cues were occluded (see below).

The additional group of unoperated turtles (data not shown in Fig. 2), which were trained in a control procedure to find reward in one of two possible unsignaled goal locations that varied in a pseudorandom order across trials, performed close to chance during the training sessions (mean percentage correct, $44.72 \pm$ $\left.1.32 \% ; F_{(9,81)}=0.3282, p=0.94\right)$. Thus, the possibility that turtles trained in the place and the cue procedures might find the 


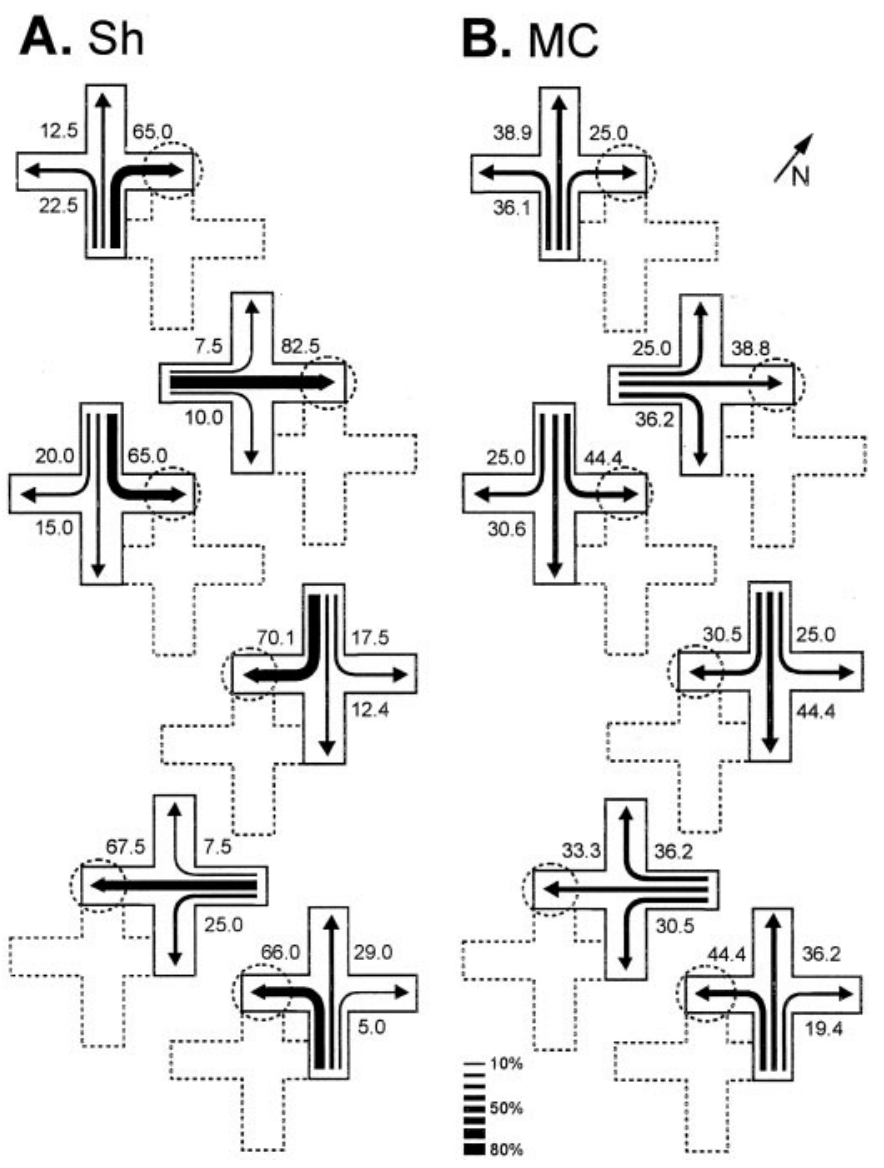

Figure 3. Trajectories chosen by sham- $(A)$ and medial cortex lesioned$(B)$ turtles in the place procedure during the transfer trials, in which the maze was displaced within the room in such a way that novel start locations, never used during training, were used. The numbers and the relative thickness of the arrows denote the percentage of times that a particular choice was made. The position of the maze during training trials is shown by dotted lines. The dotted circles show the goal location during training. The diagonal arrow and $N$ at the top right indicate north.

reward by direct vision, odor cues, or other uncontrolled variables can be disregarded.

\section{Transfer and probe tests}

During TTs, in which new start positions were used, a significant effect of the lesion appeared $\left(t_{(17)}=4.28, p=0.001\right)$ (Fig. $\left.2 A\right)$. Figure 3 shows the trajectories chosen by Sh and MC turtles in the place procedure during the TTs, in which the maze was displaced such that novel start locations, never used during training, were used. Note that, in these TTs, the Sh turtles accurately navigated toward the place in the room where the reward was located during training trials, regardless of start position and trajectory to the goal $\left(F_{(2,18)}\right.$ values $>11.47, p$ values $\left.<0.001\right)$. In the Sh group, no statistically significant differences were found in the number of place choices to the trained goal between the different transfer trials. Thus, these animals showed a preference for the goal place, regardless of the start position $\left(F_{(2,18)}\right.$ values $>11.5, p$ values $<$ 0.001 ) (Fig. $3 A$ ). In contrast, the MC turtles trained in the same procedure were unable to do so. The TT results showed that the MC turtles trained in the place task navigated at an aimless-like manner every time a new start position was used, given that the pattern of choices did not differ from a random distribution $\left(F_{(2,16)}\right.$ values $<1.54, p$ values $\left.>0.25\right)$ (Figs. $\left.2 A, 3 B\right)$.
Probe trials were conducted to analyze the relevance of the extramaze visual cues for the solution of the place task. In PT1, in which every extramaze visual cue was eliminated by surrounding the entire maze with a gray curtain, the accuracy decreased to chance level $\left(\mathrm{Sh}, t_{(9)}=0.31, p=0.75 ; \mathrm{MC}, t_{(8)}=1.41, p=0.195\right)$ (Fig. 2A), and the performance of both groups did not differ $\left(t_{(17)}=1.08, p=0.29\right)$. In PT3, when the visual cues opposite to goal location were excluded, the MC and Sh turtles showed a significant preference for the trajectory to the place at which reward was available during training trials ( $\mathrm{Sh}, t_{(9)}=4.99, p=$ 0.001; $\mathrm{MC}, t_{(8)}=4.43, p=0.002$ ) (Fig. $2 A$ ), and no differences were found between both groups $\left(t_{(17)}=1.23, p=0.23\right)$. However, a significant effect of the lesion was observed in PT2, in which only those extramaze cues placed in the proximity to the goal were occluded $\left(t_{(17)}=2.41, p=0.027\right)$. Thus, whereas the Sh turtles successfully located the goal despite the elimination of any subset of the extramaze cues $\left(t_{(9)}=4.74, p=0.001\right)$, the MC turtles failed when the extramaze visual cues placed in the proximity of the goal were concealed $\left(t_{(8)}=0.35, p=0.72\right)($ Fig. $2 A)$.

\section{Cue learning and MC lesion}

Figure $2 B$ shows the learning curve of the turtles trained in the cue procedure in which the goal location, which varied across trials, was always signaled by a conspicuous intramaze visual cue. No deficits were observed in the performance of the MC turtles relative to the $\mathrm{Sh}$ animals in the cue task. Indeed, the MC animals reached criterion faster than Sh animals $\left(F_{(1,17)}=10.8, p=\right.$ $0.004)$, although no differences were observed in the accuracy level during postcriterion trials $\left(t_{(17)}=1.55, p=0.26\right)($ Fig. $2 B)$.

\section{Probe and transfer tests}

The results of probe and transfer trials showed that the turtles trained in the cue procedure consistently selected the trajectory leading to the maze arm containing the intramaze visual cue that signaled the goal during training trials, regardless of the place at which the goal was located and whether or not the distal visual cues were available. Thus, the complete occlusion of the extramaze visual cues (PT1) did not impair the performance of any group $\left(\mathrm{Sh}, t_{(9)}=0.56, p=0.58 ; \mathrm{MC}, t_{(8)}=1.9, p=0.93\right)$. In fact, no differences were observed between $\mathrm{MC}$ and Sh turtles in any of the three types of distal visual cues occlusion tests: PT1, PT2, and PT3 $\left(F_{(2,34)}=0.31, p=0.73\right)$ (Fig. $\left.2 B\right)$. In contrast, the removal of the single intramaze visual cue associated to the goal (PT4) was sufficient to disrupt the performance of both groups $\left(\mathrm{Sh}, t_{(9)}=2.67, p=0.26 ; \mathrm{MC}, t_{(8)}=3.32, p=0.01\right)$, and the choices did not differ from chance in these probe tests (Sh, $t_{(9)}=$ $0.55, p=0.59 ; \mathrm{MC}, t_{(8)}=0.28, p=0.78$ ) (Fig. $2 B$ ). Also, during the transfer trials in which novel start positions were used, the animals consistently chose the arm signaled by the intramaze cue, regardless of both start position and trajectory to the goal $\left(\mathrm{Sh}, t_{(9)}=5.46, p<0.001 ; \mathrm{MC}, t_{(8)}=6.42, p<0.001\right)($ Fig. 2B).

\section{Histology}

Maximal and minimal extents of medial cortex lesions are represented diagramatically in Figure 4. Lesioned turtles had between 55 and $85 \%$ medial cortex removal. Performance of the lesioned turtles did not vary with the extent of medial cortex damage. Slight damage to the dorsal cortex and the dorsal ventricular ridge was observed in two animals and was not related to performance. In addition, the histological analysis did not reveal any cerebral damage in the Sh animals. 


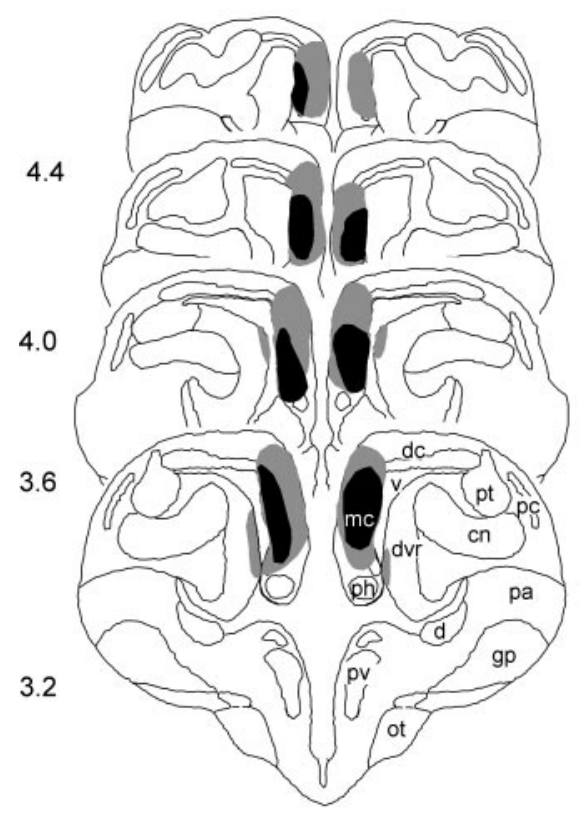

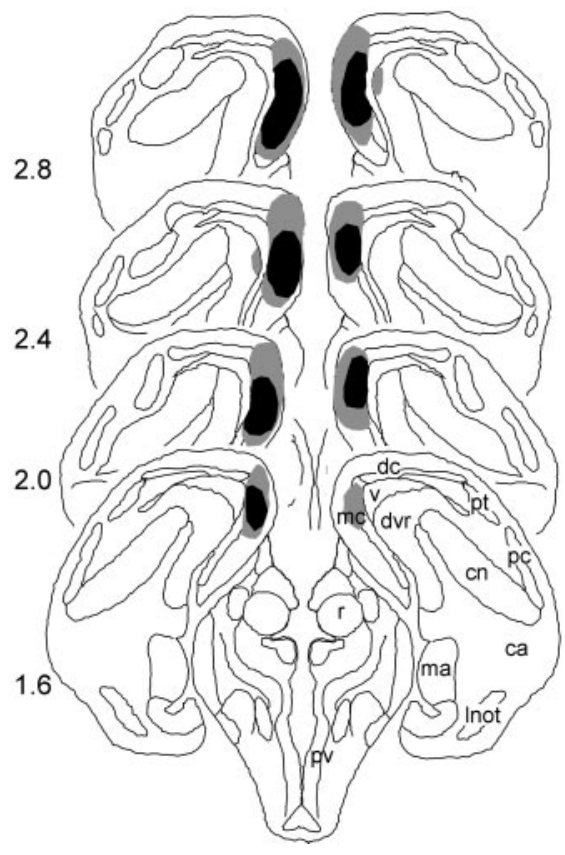

Figure 4. The largest (gray shading) and smallest (black shading) medial cortex lesions in turtles reconstructed in coronal sections according to the atlas of Powers and Reiner (1980). ca, Central nucleus of the amygdala; $c n$, core nucleus of dvr; $d$, area $\mathrm{d} ; d c$, dorsal cortex; $d v r$, dorsal ventricular ridge; $g p$, globus pallidus; Inot, lateral nucleus of the olfactory tract; $m a$, medial nucleus of the amygdala; $m c$, medial cortex; ot, optic tract; $p a$, paleostriatum augmentatum; $p c$, pyriform cortex; $p h$, primordium hippocampi; $p v$, periventricular nucleus of the hypothalamus; $p t$, pallial thickening; $r$, nucleus rotundus; $v$, ventricle.

\section{Experiment 2}

Place learning and LP lesion

Goldfish trained in the place task learned to navigate accurately to the goal. The performance of each lesion group during presurgery and postsurgery training is shown in Figure $5 A$. No significant differences in any behavioral measure were observed between the counterbalanced conditions within each group during presurgery and postsurgery training ( $F$ values $<4.2$, $p$ values $>0.08)$; therefore, these data were collapsed to calculate group percentages. The percentage of correct trials remained near chance levels for the first sessions of the experiment (all $\chi_{(1)}^{2}<3.24, p$ values $\left.>0.072\right)$. On subsequent sessions, every group progressively improved their accuracy until they reached the learning criterion. No differences in the learning rate of the different groups were observed during presurgery training $\left(F_{(4,42)}=1.63, p=0.184\right)$ (Fig. $\left.5 A\right)$. As in experiment 1 , the control group of unoperated goldfish (data not shown in Fig. 5) trained in a random procedure failed to find the reward and performed close to chance across the training sessions (mean percentage correct, $\left.50.12 \pm 2.08 \% ; F_{(6,42)}=0.47, p=0.82\right)$. Thus, the possibility that goldfish in the place task might find the reward by direct vision, odor cues, or other uncontrolled variables can be disregarded.

After surgery, training was resumed for 12 retraining sessions. During these sessions, ANOVA revealed a significant effect of the group factor $\left(F_{(3,126)}=3.35, p<0.021\right)$. Post hoc Tukey's test revealed a significantly poor performance for the goldfish with LP and Tel lesions (LP vs DP, MP, and Sh, $p$ values $<0.001$; Tel vs $\mathrm{DP}, \mathrm{MP}$, and Sh, $p$ values $<0.001)$. The lesion to the lateral pallium produced an impairment as severe as the complete ablation of the telencephalon $\left(F_{(1,19)}=1.05, p=0.31\right)$ (Fig. 5A). Indeed, during postsurgery training, the performance of the LP and Tel groups did not differ from the performance of the animals trained in the control procedure $\left(F_{(6,78)}=0.66, p=0.67\right)$. In contrast, no effect of the lesion was observed in the MP, DP, and Sh groups $\left(F_{(2,23)}=0.63, p=0.55\right)$ (Fig. $\left.5 A\right)$.

\section{Transfer tests}

Transfer tests conducted during postcriterion presurgery training showed that the goldfish were able to spontaneously choose the appropriate trajectory toward the goal when new start positions were used (Fig. $5 A$, white bars). In fact, animals in all of the groups showed a preference for the goal place, regardless of the start position $(F$ values $>4.81, p$ values $<0.026)$. No statistically significant differences were found between the different groups in the number of place responses during the presurgery transfer trials $\left(F_{(4,42)}=0.076, p=0.98\right)$.

In contrast, remarkable differences were observed in the performance of the different groups during postsurgery transfer trials. A significant effect of the lesion was found in LP and Tel animals. The postsurgery percentage of choice of the arm leading to the goal place decreased significantly in LP and Tel groups relative to presurgery performance $\left(\mathrm{LP}, t_{(10)}=4.18, p<0.002\right.$; Tel, $\left.t_{(9)}=4.83, p<0.001\right)$ and relative to the Sh, MP, and DP groups (all $p$ values $<0.009$ ). No statistically significant differences were observed between the LP and Tel groups $\left(t_{(19)}=0.36\right.$, $p=0.72$ ). In fact, both groups showed a severe impairment in their ability to locate the goal from new start places (Figs. $5 A$, black bars, 6). Interestingly, goldfish in the MP, DP, and Sh groups accurately navigated toward the goal place from each new start location after surgery and did not present statistically significant differences relative to presurgery performance $(t$ values $>$ $0.13, p$ values $>0.38)$. Similarly, no significant between-group differences were observed between MP, DP, and Sh animals in the TTs $\left(F_{(2,23)}=96.89, p=0.75\right)($ Fig. $5 A)$.

\section{Cue learning and LP lesion}

After training in the place task, goldfish were trained in a cuelearning task in a different maze and room. Animals in all of the lesion groups accurately learned the cue task, and no betweengroup differences were observed $\left(F_{(12,126)}=1.54, p=0.11\right)$ (Fig. $5 B)$. At the onset of training, during the initial 10 trials of session 1 , the choices of the animals from all of the groups were distributed at random between the goal door signaled by the intramaze 

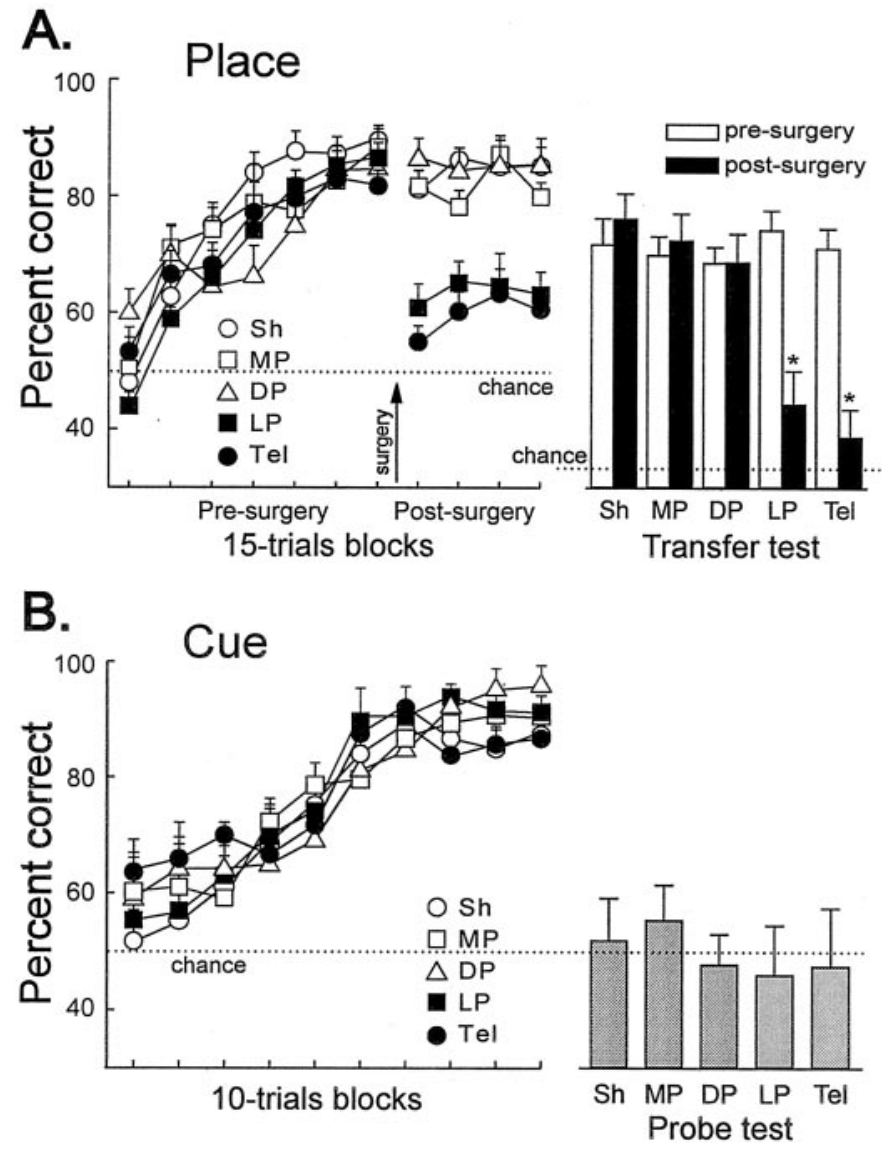

Figure 5. Spatial memory deficits after lateral pallium lesion in goldfish. $A$, Mean \pm SEM percentage of correct choices during presurgery and postsurgery training and transfer trials for each group, in the place task. At the right is shown the percentage of place responses during transfer trials in which the maze was displaced within the room, in such a way that start locations, never used during training, were used. $B$, Mean \pm SEM percentages of correct responses during training in the cue procedure. At the right is shown the percentage of correct responses in the cue removal test (probe test). ${ }^{*} p<0.05$.

visual cue and the incorrect door $\left(\right.$ all $\chi_{(1)}^{2}<0.78, p$ values $>$ 0.07 ). With training, goldfish in all of the lesion groups improved their accuracy until they reached the learning criterion. No differences in the learning rate of the different groups were observed through the training sessions $\left(F_{(12,126)}=1.54, p=0.117\right.$ ) (Fig. $5 B)$. At the end of the experiment, every group failed to find the goal in a cue removal test ( $t$ values $>2.54, p$ values $<0.038$ ) (Fig. $5 B)$. The results of this test indicate that the fish did not perceive the glass barrier that blocked the incorrect door and that the sole relevant information to solve the task was that provided by the cue signaling directly the goal.

\section{Histology}

Histological analysis of the goldfish brain showed that the lesions were homogeneous in size and location (Fig. 7). LP goldfish had between 64 and $89 \%$ lateral pallium removal, DP animals had between 56 and $85 \%$ dorsal pallium removal, and finally the MP goldfish had between 71 and $93 \%$ medial pallium removal. According to the nomenclature of Nieuwenhuys (1963), the LP lesions damaged the ventral part of the lateral zone of the area dorsalis (Dlv). The posterior zone of the area dorsalis (Dp) and the most ventral part of the lateral division of the area dorsalis
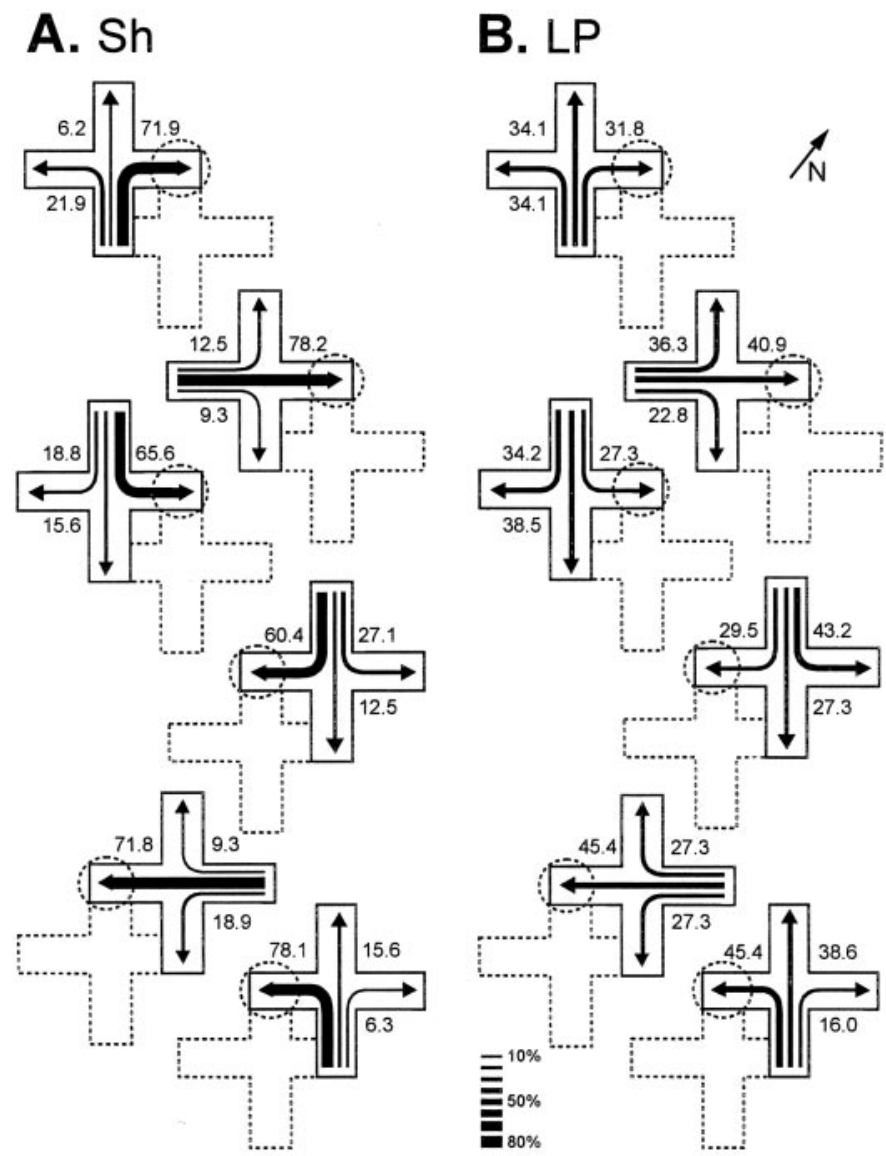

Figure 6. Trajectories chosen by sham- $(A)$ and lateral pallium lesioned$(B)$ goldfish during the transfer trials conducted after surgery. The numbers and the relative thickness of the arrows denote the percentage of times that a particular choice was made. The position of the maze during training trials is shown by dotted lines. The dotted circles show the goal location during training. The diagonal arrow and $N$ at the top right indicate north.

(Dld) appeared damaged in some animals. Dorsal zone of the area dorsalis (Dd) lesions included most of the dorsal division of the area dorsalis pars medialis (Dmd) and Dd areas, as well as the most dorsal part of Dld. The MP lesions damaged, nearly completely, the ventral region of area dorsalis pars medialis (Dmv), affecting in some cases the most medial portion of the Dmd area. The area dorsalis pars centralis (Dc) and the subpallial area ventralis pars dorsalis (Vd) appeared intact or minimally damaged. Telencephalon-ablated brains exhibited complete telencephalic tissue removal in all of the animals, whereas preoptic and hypothalamic areas and optic tracts were entirely spared.

\section{DISCUSSION}

The most noteworthy result of the experiments reported here is that, similar to the mammalian and avian hippocampus, the reptilian medial cortex and the lateral telencephalic pallium of the teleost fish are both selectively involved in spatial memory. As discussed below, these results have considerable comparative value and provide interesting insights about the evolution of the vertebrate forebrain. In addition, present functional data could contribute significantly to clarify the identity of the pallial zones in ray-finned fishes, long obscured during the past century. 

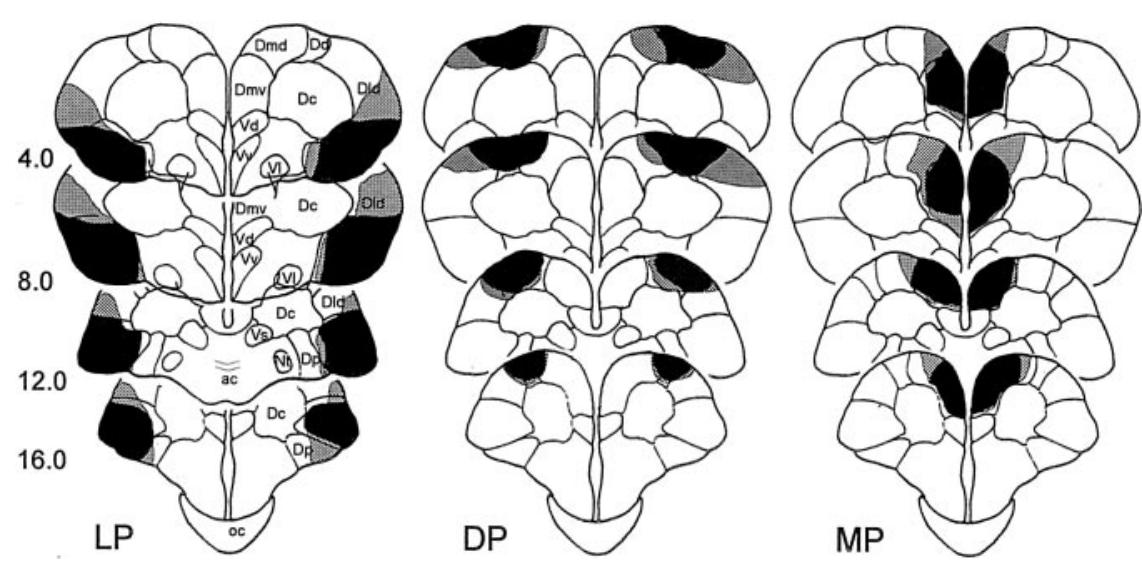

Figure 7. The largest (gray shading) and smallest (black shading) extent of the LP, DP, and MP lesions in goldfish reconstructed in coronal sections according to the atlas of Peter and Gill (1975). $a c$, Anterior commissure; $N t$, nucleus taeniae; $o c$, optic chiasm; $V$, area ventralis; $V l$, area ventralis pars lateralis; $V s$, area ventralis pars supracommissuralis; $V v$, area ventralis pars ventralis.

\section{Medial cortex and place learning in turtles}

In the first experiment, turtles with lesions to the MC or shamoperated animals were trained in a place procedure. Although all of the turtles learned to reach the goal during training trials, the probe and transfer tests revealed that the MC lesion produced a clearly defined place-learning deficit. The MC turtles were unable to navigate using the distributed array of extramaze visual cues. Whereas the Sh turtles successfully located the goal despite the elimination of any subset of the extramaze cues, the MC turtles failed when the extramaze visual cues placed in the proximity of the goal were concealed (Fig. $2 A$ ). In addition, these animals failed to reach the goal location from novel start positions, when navigation to the goal involved the use of completely new path trajectories, as Sh turtles accurately did (Fig. 3). The ability of the Sh turtles to spontaneously use new routes or shortcuts without previous reinforcing experience and to navigate directly to the goal regardless of partial losses of relevant environmental information indicate that these animals have encoded the spatial relationships among the various extramaze cues and the goal in a map-like allocentric representation (López et al., 2000c, 2001). In contrast, the MC turtles trained in the same procedure solved the task by means of approaching a single distal visual cue or retinal snapshot that they associated with the goal (López et al., 2000c). These data provide strong evidence regarding a cognitive mapping function of the medial cortex in turtles.

In addition, the data of the MC and Sh turtles in the cue procedure, in which the goal location varied across trials but was always directly signaled by a conspicuous visual cue, indicate that the impairment shown by the MC turtles in the place task was not a general learning impairment but was specifically related to allocentric spatial memory. That is, like hippocampus-lesioned mammals and birds, the MC turtles showed no deficit relative to the Sh animals in the cue task (Fig. 2B); indeed, the MC turtles learned this task faster. The probe and transfer tests revealed that the turtles solved this task on the basis of a guidance strategy, and the MC lesions did not impair this kind of representation (for other tasks not impaired by MC lesions in reptiles, see Powers, 1990). Thus, the effects of MC lesion in turtles reported here are closely similar to those after hippocampal damage in mammals and birds trained in comparable procedures, not only in terms of the tasks that are impaired but also in those that are not impaired (Morris et al., 1982; Jarrard, 1983; McDonald and White, 1993; Good and Macphail, 1994; Hampton and Shettleworth, 1996; Fremouw et al., 1997) or even facilitated (Eichenbaum et al., 1988; M'Harzi and Jarrard, 1992; White and McDonald, 1993; Strasser and Bingman, 1997).
The present results indicate that the reptilian medial cortex, considered homologous to the hippocampal formation of mammals and birds on basis of anatomical and physiological evidence (Northcutt, 1981; Ulinski, 1990; Nieuwenhuys et al., 1998), also share a central role in spatial cognition. The medial cortexdependent allocentric spatial learning and memory capabilities in reptiles closely parallel the hippocampal spatial memory system described in mammals and birds, suggesting that such a medial pallium-dependent memory system could be a primitive feature in amniotes, that is, could have been present already in the common reptilian ancestor of modern reptiles, mammals, and birds that inhabited the earth in the Mesozoic era.

\section{Lateral pallium and place learning in goldfish}

In the second experiment, we analyzed the involvement of particular subdivisions of the telencephalic pallium of the goldfish in spatial memory. The fish learned the place task with accuracy, and the results of the transfer tests showed that these animals are able to spontaneously choose the appropriate trajectory toward the goal from novel start places, indicating mapping abilities (Fig. 5A) (Rodríguez et al., 1994; Salas et al., 1996b; López et al., 2000a). Interestingly, goldfish with LP lesions showed a dramatic and selective place-memory impairment. Thus, after surgery, the performance of the LP animals decreased nearly to chance (Fig. $5 A$ ). In addition, during postsurgery transfer tests, LP goldfish showed a severe impairment in their ability to locate the goal from new start places (Figs. $5 A, 6$ ), revealing a profound spatial cognition deficit. Indeed, the deficit shown by LP goldfish during postsurgery training and transfer trials was as severe as that of the Tel animals. In contrast, MP and DP lesions, like Sh operations, produced no observable deficit in the ability of goldfish to navigate to goal location. Furthermore, LP- and Tel-lesioned goldfish accurately mastered the cue-learning task (Fig. $5 B$ ). These results indicate that, like the hippocampal pallium of reptiles, birds, and mammals, the lateral pallium of teleost fish is selectively involved in learning and use of map-like or relational spatial representations for allocentric navigation but is not critical for simple stimulus-response associations or cue learning. Therefore, these results demonstrate a striking functional similarity between the lateral telencephalic pallium of teleost fish and the hippocampal pallium of amniotes, providing compelling evidence for an eversion process in the formation of the teleost telencephalon with considerable preservation of the original topology. The hypothesis of eversion concerning the embrionary development of the telencephalon in ray-finned fishes implies the reversal of the medial-to-lateral topography observed in the evaginated telen- 
cephalon (Nieuwenhuys, 1963; Northcutt and Braford, 1980). Thus, the developmentally medial (hippocampal) pallium is predicted to lie laterally in everted telencephalons. That is, the Dlv is considered homologous to the medial pallium of land vertebrates, although more disagreement still exists about the homology of the Dp (Northcutt and Braford, 1980; Northcutt, 1995; Butler, 2000). In addition, on the base of topological, embryological, neurochemical, and connectivity evidence, the Dmd is considered homologous to the pallial amygdala, whereas the Dd and the Dld correspond to the dorsal pallium of amniotes (Northcutt and Braford, 1980; Nieuwenhuys and Meek, 1990; Braford, 1995; Butler, 2000). Also in agreement with the present results, neither the pallial amygdala nor the dorsal pallium or isocortex have an essential role in spatial learning and memory (Leonard and McNaughton, 1990; Peinado-Manzano, 1990; Gaffan and Gaffan, 1991; Zola-Morgan et al., 1994). Thus, the present functional data converge with developmental and neuroanatomical evidence, significantly contributing to clarify the identity of the pallial zones in ray-finned fishes.

\section{Implications for the evolution of the vertebrate pallial forebrain}

The present results suggest that the forebrain of vertebrates, whether everted or evaginated, contains a form of map-like or relational spatial memory system characterized by representational flexibility (O'Keefe and Nadel, 1978; Cohen and Eichenbaum, 1994) that is critically dependent on the pallial areas homologous to the mammalian hippocampus. Modern mammals, birds, reptiles, and ray-finned fishes share a common evolutionary ancestor that lived some 400 million of years ago (Carroll, 1988). For characters such as brain and behavior, for which there is little evidence in the fossil record, any hypothesis of homology must be inferred from the distribution of characters observed in extant species on the principle of parsimony (Wiley, 1981; Northcutt, 1995). In this regard, the close functional similarity among the hippocampus of mammals and birds, the medial cortex of reptiles, and the lateral telencephalic pallium of teleost fish suggests that, early in vertebrate evolution, the medial pallium in an ancestral fish group, from which these extant vertebrate groups evolved, became specialized as a navigational device for processing spatial information that could be encoded as a map-like or relational memory representation of the environmental space. This primitive feature seems to have been conserved through the evolution of these vertebrate lineages, although the structure itself appears to have undergone major morphological changes.

\section{REFERENCES}

Bingman VP, Riters LV, Strasser R, Gagliardo A (1998) Neuroethology of avian navigation. In: Animal cognition in nature (Balda R, Pepperberg I, Kamil A, eds), pp 201-226. New York: Academic.

Braford MR (1995) Comparative aspects of forebrain organization in the ray-finned fishes: touchstones or not? Brain Behav Evol 46:259-274.

Burgess N, Jeffery KJ, O'Keefe J (1999) The hippocampal and parietal foundations of spatial cognition. London: Oxford UP.

Butler AB (2000) Topography and topology of the teleost telencephalon: a paradox resolved. Neurosci Lett 293:95-98.

Butler AB, Hodos W (1996) Comparative vertebrate neuroanatomy: evolution and adaptation. New York: Wiley.

Carroll RL (1988) Vertebrate paleontology and evolution. New York: Freeman.

Cohen HJ, Eichenbaum H (1994) Memory, amnesia, and the hippocampal system. Cambridge, MA: MIT.

Eichenbaum H, Fagan A, Mathews P, Cohen N (1988) Hippocampal system dysfunction and odor discrimination learning in rats: Impairment or facilitation depending on representational demands. Behav Neurosci 102:331-339.
Eichenbaum H, Dudchenko P, Wood E, Shapiro M, Tanila H (1999) The hippocampus, memory, and place cells: Is it spatial memory or a memory space? Neuron 23:209-226.

Fremouw T, Jackson-Smith P, Kesner RP (1997) Impaired place learning and unimpaired cue learning in hippocampal-lesioned pigeons. Behav Neurosci 111:963-975.

Gaffan D, Gaffan EA (1991) Amnesia in man following transection of the fornix. A review. Brain 114:2611-2618.

Gaffan D, Harrison S (1989) Place memory and scene memory: effects of fornix transection in the monkey. Exp Brain Res 74:202-212.

Good M, Macphail EM (1994) The avian hippocampus and short-term memory for spatial and non-spatial information. Q J Exp Psychol 47B:293-317.

Hampton RR, Shettleworth S (1996) Hippocampal lesions impair memory for location but not color in passerine birds. Behav Neurosci 110:831-835.

Jarrard LE (1983) Selective hippocampal lesions and behavior: effects of kainic acid lesions on performance of place and cue tasks. Behav Neurosci 97:873-889.

Leonard BJ, McNaughton BL (1990) Spatial representation in the rat: conceptual, behavioral and neurophysiological perspectives. In: Neurobiology of comparative cognition (Kesner RP, Olton DS, eds), pp 363-422. Hillsdale, NJ: Erlbaum.

López JC, Broglio C, Rodríguez F, Thinus-Blanc C, Salas C (1999) Multiple spatial learning strategies in goldfish (Carassius auratus). Anim Cogn 2:109-120.

López JC, Bingman VP, Rodríguez F, Gómez Y, Salas C (2000a) Dissociation of place and cue learning by telencephalic ablation in goldfish. Behav Neurosci 114:687-699.

López JC, Broglio C, Rodríguez F, Thinus-Blanc C, Salas C (2000b) Reversal learning deficit in a spatial task but not in a cued one after telencephalic ablation in goldfish. Behav Brain Res 109:91-98.

López JC, Rodríguez F, Gómez Y, Vargas JP, Broglio C, Salas C (2000c) Place and cue learning in turtles. Anim Learn Behav 28:360-372.

López JC, Gómez Y, Rodríguez F, Broglio C, Vargas JP, Salas C (2001) Spatial learning in turtles. Anim Cogn 4:49-59.

McDonald RJ, White NM (1993) A triple dissociation of memory system: hippocampus, amygdala, and dorsal striatum. Behav Neurosci 107:3-22.

M'Harzi M, Jarrard LE (1992) Strategy selection in a task with spatial and nonspatial components: effects of fimbria-fornix lesions in rats. Behav Neural Biol 58:171-180.

Morris RGM, Garrud P, Rawlins JNP, O'Keefe J (1982) Place navigation impaired in rats with hippocampal lesions. Nature 297:681-683.

Muñoz MD, Magariños-Ascone C, Gaztelu JM, García-Austt E (1998) Frequency potentiation in the medial cortex of young turtles brains in vitro. Brain Behav Evol 52:263-269.

Nieuwenhuys R (1963) The comparative anatomy of the actinopterygian forebrain. J Hirnforsch 6:171-200.

Nieuwenhuys R, Meek J (1990) The telencephalon of actinopterygian fishes. In: Comparative structure and evolution of the cerebral cortex (Jones EG, Peters A, eds), pp 31-73. New York: Plenum.

Nieuwenhuys R, ten Donkelaar HJ, Nicholson C (1998) The central nervous system of vertebrates. Berlin: Springer.

Northcutt RG (1981) Evolution of the telencephalon in nonmammals. Annu Rev Neurosci 4:301-350.

Northcutt RG (1995) The forebrain of gnathostomes: in search of a morphotype. Brain Behav Evol 46:275-318.

Northcutt RG, Braford MR (1980) New observations on the organization and evolution of the telencephalon of actinopterygian fishes. In: Comparative neurology of the telencephalon (Ebbesson SOE, ed), pp 41-98. New York: Plenum.

O'Keefe J, Nadel L (1978) The hippocampus as a cognitive map. London: Oxford UP.

Peinado-Manzano MA (1990) The role of the amygdala and the hippocampus in working memory for spatial and non-spatial information. Behav Brain Res 38:117-134.

Peter RE, Gill VE (1975) A stereotaxic atlas and technique for forebrain nuclei of the goldfish, Carassius auratus. J Comp Neurol 159:69-102.

Powers AS (1990) Brain mechanisms of learning in reptiles. In: Neurobiology of comparative cognition (Kesner RP, Olton DS, eds), pp 157-177. Hillsdale: Lawrence Erlbaum Associates.

Powers AS, Reiner A (1980) A stereotaxic atlas of the forebrain and midbrain of the eastern painted turtles (Chrysemis picta picta). J Hirnforsch 21:125-159.

Rodríguez F, Duran E, Vargas JP, Torres B, Salas C (1994) Performance of goldfish trained in allocentric and egocentric maze procedures suggests the presence of a cognitive mapping system in fishes. Anim Learn Behav 22:409-420.

Salas C, Broglio C, Rodríguez F, López JC, Portavella M, Torres B (1996a) Telencephalic ablation in goldfish impairs performance in a "spatial constancy" problem but not in a cued one. Behav Brain Res 79:193-200. 
Salas C, Rodríguez F, Vargas JP, Durán E, Torres B (1996b) Spatial learning and memory deficits after telencephalic ablation in goldfish trained in place and turn maze procedures. Behav Neurosci 110:965-980

Schwerdtfeger WK, Smeets WJAJ (1988) The forebrain of reptiles. Current concepts of structure and function. Basel: Karger.

Sherry DF, Duff SJ (1996) Behavioral and neural bases of orientation in food storing birds. J Exp Biol 199:165-172.

Sherry DF, Vaccarino AL (1989) Hippocampus and memory for food caches in black-capped chickadees. Behav Neurosci 103:308-318.

Strasser R, Bingman VP (1997) Goal recognition and hippocampal formation in the homing pigeon (Columba livia). Behav Neurosci 111:1245-1256.

Ulinski PS (1990) The cerebral cortex of reptiles. In: Cerebral cortex,
Vol 8A, Comparative structure and evolution of the cerebral cortex (Jones EG, Peters A, eds), pp 139-215. New York: Plenum.

Vargas JP, Rodríguez F, López JC, Arias JL, Salas C (2000) Spatial learning-induced increase in the argyrophilic nucleolar organizer region of dorsolateral telencephalic neurons in goldfish. Brain Res 865:77-84.

White NM, McDonald RJ (1993) Acquisition of a spatial conditioned place preference is impaired by amygdala lesions and improved by fornix lesions. Behav Brain Res 55:269-281.

Wiley EO (1981) Phylogenetics. The theory and practice of phylogenetic systems. New York: Wiley.

Zola-Morgan S, Squire LR, Ramus SJ (1994) Severity of memory impairment in monkey as a function of locus and extent of damage within the medial temporal lobe memory system. Hippocampus 4:483-495. 